To appear in Biology and Philosophy 22(5)

(C) Springer

\title{
Psychological Categories as Homologies: Lessons from Ethology
}

\author{
Marc Ereshefsky \\ Department of Philosophy \\ 2500 University Drive, NW \\ University of Calgary \\ Calgary, Alberta T2N 1N4 \\ Canada \\ Email: ereshefs@ucalgary.ca
}

\section{Key words}

adaptationism, behavioral homology, evolutionary psychology, functionalism, homology, ontogeny, phylogeny

\footnotetext{
Abstract

This paper takes up the project of studying psychological categories as homologies. Ethologists have numerous theoretical ideas concerning the phylogeny and ontogeny of behavioral homologies. They also have well-developed operational methods for testing behavioral homologies. Many of these theoretical ideas and operational criteria can be applied to psychological homologies. This paper suggests that insights from ethology should be incorporated in adaptationist and functionalist approaches to psychology. Doing so would strengthen those approaches.
} 


\section{Introduction}

The modern study of psychological categories as homologies begins with Darwin’s famous suggestion that such emotions as fear, courage, and shame are homologies in “man and the lower animals” (Richards 1987). Morgan, Baldwin, and James continued the project of studying psychological categories as homologies in the late $19^{\text {th }}$ and early $20^{\text {th }}$ Centuries (Richards 1987; Johnston 2001). In the years surrounding World War II, Lorenz and Tinbergen developed the field of ethology with its emphasis on phylogenetic analyses of behavioral and psychological traits (Johnston 2001; Alcock 2003; Burkhardt 2005). In the mid-20 $0^{\text {th }}$ Century, Lehrman (1953) steered ethology in a different direction by critiquing the nativism of early ethologists and suggesting a more careful study of the ontogeny of behavior.

In the 1970s and 1980s, ethology took a backseat to the adaptationist programmes of behavioral ecology, sociobiology, and evolutionary psychology (Alcock 2003; Bateson 2001, 2003). These programmes study psychological traits as products of evolution, but they pay little attention to the phylogeny and ontogeny of such traits. Their primary focus is determining the adaptive advantages of psychological traits (Richardson 2007). Consider, for example, evolutionary psychology’s explanations for cheater detection and mate preferences. Evolutionary psychologists approach such traits as design problems, applying a form of reverse engineering to infer the evolutionary problems those traits were selected to solve. The 1970s and 1980s also saw the rise of functionalist accounts of psychological traits in philosophy and cognitive science. Functionalists suggest that instances of a psychological category are identified by their mediating a similar pattern of 
physiological and behavioral inputs and outputs (Bickle 2006). Functionalists, like their evolutionary counterparts, are interested in the function a psychological state performs. They pay little if any attention to the phylogeny or ontogeny of such states.

Despite the rise of adaptationist and functionalist approaches to psychology, the project of studying psychological categories as homologies did not go away. In the 1980s Bateson and other biologists argued for the return of ethological work in psychology and behavioral studies, and the 1990s saw a resurgence of investigating behaviors as homologies (Greene 1999; Alcock 2003; Bateson 2003). These biologists contend that adaptational approaches to behavior and psychology wrongfully neglected the phylogeny and ontogeny of such traits. Recently at least a couple of philosophers have come to a similar conclusion. Paul Griffiths $(1997,2007)$ and Mohan Matthen (1998, 2000, this issue) argue that functionalist and adaptationist approaches to psychology would be improved by focusing on the phylogeny of psychological traits.

This paper takes up the project of studying psychological categories as homologies. Ethologists have numerous theoretical ideas concerning the phylogeny and ontogeny of behavioral homologies. They also have well-developed operational methods for testing behavioral homologies. This paper explores which theoretical ideas and operational criteria from ethology can be applied to psychological homologies. In broader strokes, some psychologists, biologists, and philosophers adopt an adaptationist approach to psychological categories. Others adopt a functionalist approach. As we shall see, applying insights from ethology to functionalist and adaptationist accounts of psychology strengthens those accounts. 


\section{Behavioral Homology}

Hierarchy

When ethologists talk about behavioral homologies, they talk about a broad range of phenomena, from web building by spiders to courtship rituals in non-human primates. Traditionally, behavioral homologies were tied to morphological homologies.

Biologists thought that behavioral homologies were too variable for consistent identification and too ephemeral for rigorous testing. Consequently, biologists identified behavioral homologies by their corresponding morphological structures and movements (Lauder 1994; Greene 1994). Though Lorenz and Tinbergen homologized nonmorphological characters in the first part of the $20^{\text {th }}$ Century, the view that behavioral homologies require corresponding morphological characters remained prevalent through the 1980s (Lauder 1994). In the last 30 years ethologists have dropped the requirement that a behavioral homology be identified with a corresponding morphological structure or movement (Wenzel 1992; Lauder 1994; Greene 1994). Only recently has behavior come into its own right.

The idea that a behavioral homology need not have a particular structural basis gives rise to a hierarchical approach to behavioral homology. This approach is part of a more general view concerning the hierarchical nature of homology (Brigandt, this issue). According to that view, whether a trait is a homology depends on the level of biological organization being examined (Hall 1994). For example, humans and chickens have homologous morphological digits caused by non-homologous developmental processes (Hall 2003: 417). A common explanation for such hierarchical relations is the substitution of new genes in the gene networks underlying the derived states of a 
morphological homology (Abouheif 1999). The primitive and derived states of a morphological homology are caused by different sets of genes (Striedter and Northcutt 1991: 183). The hierarchical relation between homologous and non-homologous states goes the other way too. Homologous genes and development processes cause nonhomologous phenotypes. For example, the homologous gene Pax-6 initiates the development of non-homologous eyes in mammals and flies (Wagner 2001).

Similar hierarchical features occur in behavioral homologies (Wenzel 1992; Lauder 1994). Striedter and Northcutt (1991: 185-6) cite the case of grasshopper species that produce homologous songs using different morphological patterns. In one species, songs are produced by an insect rubbing its forewings with its legs; in another species, songs are produced by an insect rubbing its legs with its mandibles. Striedter and Northcutt (ibid.) suggest that as grasshopper songs evolved, the morphological substrates for such songs diverged. Just as new genes may contribute to an old morphological homology, new morphological parts or movements may contribute to an old behavioral homology. There are also cases of homologous morphological elements underlying nonhomologous behaviors (Wenzel 1992: 365; Lauder 1994: 170ff). Lauder (1994: 172) writes of "homologous patterns of muscle activity" that give rise to non-homologous behaviors. Wenzel (1992: 365) suggests that this phenomenon "may be common because behaviors serving other purposes will have to be laid over anatomy and motor patterns defined for primary functions.”

Two points concerning the hierarchical nature of behavioral homology are worth keeping in mind. First, behavioral homologies need not be reduced to their physical substructures, for the same behavioral homology may be the result of different 
morphological structures and movements. Second, and closely related, instances of a behavioral homology can vary greatly. The assertion that a trait can vary is almost a truism in biology. Grasshopper songs, tail wagging motions in courtship rituals, and a myriad of other behaviors vary. The hierarchical nature of behavioral homology helps explain such variation. Why, for example, are different tail wagging motions of Tilapia species assumed to be the same behavioral homology (Wenzel 1992: 366)? There is the operational answer concerning how ethologists identify different motions as the same behavioral homology. We will get to that answer shortly. Then there is the theoretical answer that different motions are instances of the same behavioral homology because they share a unique phylogeny. As Müller (2003) and Ghiselin (2005) assert, homology is a relation of sameness (or identity) and not qualitative similarity. (As Brigandt's [this issue] explains, the same homologous character can have different character states.) Here we have a tidy explanation why the different tail wagging motions of Tilapia are variable yet instances of the same trait. Different underlying ontogenetic factors and morphological substrate result in the same behavior because those behaviors are instances of the same phylogenetic (homologous) unit. Phylogeny is essential for identifying behavioral variants as the same behavior. In the next subsection we see that studying ontogeny is vital for explaining similarity and difference among those variants.

\section{Ontogeny}

Biologists offer a number of proposals for defining 'homology' (Hall 1994; Bock and Cardew 1999). Arguably, there are two main approaches to homology: the taxonomic approach and the developmental approach. The taxonomic approach, rooted in 
phylogenetic systematics, defines two traits as homologous when they are derived from a common ancestor. The developmental approach, stemming from developmental biology, focuses on the ontogeny of homologous traits within and among organisms (Brigandt 2003). The debate over the correct definition of 'homology' is somewhat polarizing, with some biologists choosing one approach to the exclusion of the other (for example, Cracraft 2005; for further discussion, see Brigandt, this issue and Griffiths, this issue). Despite such disagreements, contemporary ethologists investigate both the phylogeny and the ontogeny of behavioral homologies. Historically, the founders of ethology (Lorenz and Tinbergen) focused on the taxonomic aspect of behavioral homology. In the 1950s Lehrman suggested incorporating an ontogenetic approach to behavioral homology (Johnston 2001; Alcock 2003; Burkhardt 2005). The majority of ethologists of the last thirty years promote a combined taxonomic-developmental approach to behavioral homology (Wenzel 1992; Lauder 1994; Greene 1994; Bateson 2001; Gottlieb 2001).

Before focusing on the ontogeny of behavioral homology, it is useful to review some recent work in developmental biology on the ontogeny of homology. A common theme is that morphological homologies are caused by developmental modules (Raff 1996; Wagner 1996, 1999, 2001; Brigandt, this issue). Developmental modules are relatively independent sets of developmental constrains that allow for the stable production of homologies. Developmental modules are sufficiently dissociated so that a breakdown or change in one module need not adversely affect the development of other modules. Such modules are seen as crucial for the existence of reoccurring phenotypic units (that is, homologies). They are also seen as important for phenotypic plasticity. Such plasticity comes in two forms. Environmental differences and genetic changes 
cause variation among instances of a homologue. A homologue's developmental module limits that variation. Wagner (1999) refers to the stable variation found among instances of a homologue as "shared variational properties." Plasticity of the second form is the evolution of new phenotypes through the duplication of preexisting modules (Raff 1996). There is plenty of evidence that key morphological features are the result of the multiplication of independent developmental modules (Carroll 2005).

A similar form of modularity has been posited for behavioral homologies (Wagner 1999; Bateson 2003). Developmental modules for behavioral homologies are thought to be dissociated from other such modules. Wagner (1999: 183), for example, writes that the developmental modules underlying behavioral homology "are selfregulatory and independent of the context in which they occur.” As evidence for such modularity, Bateson (2003: 12-13) reports experiments showing dissociation between a chick’s ability to imprint and a chick's ability to learn visual discrimination. The experiments involve selectively administering surgical lesions to areas of a chick’s brain associated with these activities. Bateson reports that a lesion in one area does not adversely affect the development of a behavior associated with the other area. He concludes that such behaviors are neurologically modulated. There is much speculation on the nature of developmental modules for morphological and behavioral traits. The developmental modules underlying morphological homology are thought to be gene regulatory networks (Abouheif 1999; Wagner 2007) The developmental modules underlying behavioral homologies are thought to be neural nets (Bateson 2003: 12). Just as in the case of morphological homology, plasticity is seen as an important aspect of behavioral homology, and that plasticity is thought to be the result of 
developmental modularity. Gottlieb (2001) and Bateson (2003, 2004) write that there is selection for organisms with developmental modules that allow for variable developmental responses depending on the environment. There is, as Bateson (2004: 38) describes it, selection for modules that "increase plasticity and behavioral diversity" (Bateson 2004, 38). Ethologists frequently emphasize the developmental canalization of behavioral variants, citing, for instance, the various anti-predator behaviors used by snakes (Greene 1994: 377) and the different kinship recognition songs employed by birds (Wenzel 1992: 375).

The literature on the ontogeny of behavioral homology often contrasts developmental constraints from their accompanying triggers. Yet one might wonder how constraints and triggers are distinguished. Inheritance does not distinguish between the triggers and constraints that cause behavior. If 'inheritance' merely means the stable replication of resources, then both constraints and triggers are inherited. Constraints such as gene regulatory networks and neurological networks are replicated, but so are environmental triggers such as conspecific calls and exposure to certain classes of predators. Though inheritance may not distinguish constraints and triggers, ethologists tend to assume that constraints are less variable and more stable than triggers. Furthermore, they assume that constraints are internal to an organism (gene or neurological networks), while triggers are external to an organism (environmental factors or other organisms).

At first glance, one might think that constraints and triggers, as causes of behavior, line up with the distinction between innate and acquired (or learned) behaviors. Early ethologists, namely Lorenz and Tinbergen, thought that behaviors were innate in a 
genetic sense (Lehrman 1953; Johnston 2001; Alcock 2003). Environmental stimuli merely ‘release behavior' or 'unblock' instinctive behavior patterns (Burkhardt 2005: 171, 372). In the last 20 years, the nativist school in ethology has fallen on hard times. Few contemporary ethologists label a behavior as innate or acquired (Wenzel 1992: 376; Alcock 2003: 7). As Alcock (ibid.) notes, the instinct-learning distinction "evaporated" from behavioral biology texts by the 1990s. Why have ethologists dropped the innateacquired distinction? They see behavior as the result of the interaction of both internal and external factors. As Wenzel (1992: 375) writes, "thorough studies of ontogeny of behavior show continual interaction" between the two. Though contemporary ethologists do distinguish between constraints and triggers that underlie behaviors, very few of them talk of behaviors being innate or acquired.

The overturning of nativism and the abandonment of the innate-acquired distinction within ethology is largely credited to Lehrman’s (1953) work (Johnston 2001; Bateson 2003). Lehrman criticized the nativist inference that if a behavior is stable across generations then that behavior is innate and determined by genes. Lehrman observed that other factors besides genetic ones cause the stable reproduction of behaviors. He also critiqued nativist theories by suggesting that the ontogeny of a behavioral trait is due to the continual interaction of an organism and its environment. Two aspects of this interaction are worth highlighting. First, development is not the single operation of genes interacting with environmental resources, as often envisioned by nativists, but a sequence of interactions building on the products of previous interactions (Lehrman 1953: 31). Second, the interactions themselves are not between genes and the environment, as envisioned by nativists, but between gene products 
(prezygotic or postzygotic) and the environment (Lehrman ibid.; Bateson 2003: 12).

Contemporary ethologists have wholeheartedly accepted Lehrman's critique of nativism and his rejection of the innate-acquired distinction (Bateson 2001).

This subsection began by noting that early ethologists such as Lorenz and Tinbergen believed that behavior was innate and that the aim of ethology was to develop a phylogenetic theory of behavior. Contemporary ethologists have abandoned nativism and now emphasize both the phylogeny and ontogeny of behavior. Contemporary ethologists employ a taxonomic-developmental (or an evolutionary-developmental) approach to behavior.

\section{Operational Criteria}

Let us turn to the operational criteria used for identifying and testing behavioral homology. The importance of having operational tests for behavioral homology is twofold. First, there is the question of whether a putative behavioral homology is indeed a homology rather than a homoplasy. Second, operational criteria provide methods for testing adaptational hypotheses concerning behavior. As many biologists and philosophers point out, a proper test of an adaptational hypothesis requires that the hypothesis be tested historically (Burian 1992; Griffiths 1996). By restricting adaptational hypotheses to those that posit homologies, we can subject adaptive hypotheses to an array of phylogenetic tests. This is true not only of hypotheses that explicitly concern homologies, but also those that posit analogies. Analogies, after all, are collections of homologies (for example, the analogy class wing contains four distinct homologies: bird wing, bat wing, pterosaur wing, and insect wing). An insight of early 
ethologists (Lorenz and Tinbergen) is that a behavior should be identified as a homology before positing an adaptational hypothesis concerning that trait (Burkhardt 2005).

Before turning to operational criteria in ethology, it is useful to give an overview of the various phylogenetic tests biologists use for identifying homologies and testing adaptational hypotheses concerning homologies. One set of tests establishes whether a trait is a homology rather than a homoplasy (a similarity not due to common ancestry). A second set of tests determine the ancestral and derived states of a homology - what biologists call 'trait polarity.' Both sets of tests determine the phylogeny of a trait. They are also useful for testing adaptational hypotheses because they test an adaptive scenario against evidence of a trait's phylogeny. A third set of tests, often called 'comparative methods,' also compares adaptive scenarios with phylogenetic information (Sterelny and Griffiths 1999: 243ff). One type of comparative test uses biogeography to see if a derived state is fitter than its primitive state in relevant past environments. Another type of comparative method tests whether the sequence of evolutionary change posited by an adaptive scenario matches phylogenetic data concerning that change (Griffiths 1996: 522). A third type of comparative method tests an adaptive scenario by comparing a trait's posited phylogeny against the phylogenies of other relevant traits (ibid.: 523). (For example, if bears are thought to have low birth weights because such weights confer an adaptive advantage during hibernation, we should test if low birth weight evolved after the evolution of hibernation.)

Ethologists of the last twenty years have developed a number of methods for testing and identifying behavioral homologies. One motivation for developing such methods has been to answer critics of behavioral homology (Greene 1994; Lauder 1994). 
Such critics question both the existence of behavioral homologies and our ability to identify behaviors as homologies. The first worry is that if behaviors are often learned, then such behaviors are homoplastic and not homologous (Greene 1994: 379; Lauder 1994: 178). The second worry questions whether behaviors are stable enough for empirical testing and cites "the evanescence of each behavioral act” (Greene 1994: 377). Supporters of behavioral homology respond that "the bones that morphologists actually measure are usually no more than inanimate, desiccated shadows of what they once were” (Greene 1999: 176).

In a more positive vein, ethologists develop operational criteria for identifying and testing behavioral homologies. Three such criteria are ethological versions of Adolf Remane’s (1952) classic criteria for identifying morphological homology (Wenzel 1992, 1993; Lauder 1994). Remane’s criteria are physical position, special quality (the distinctiveness and complexity of a character), and connection to evolutionary intermediates. Ethologists apply these criteria to behavior, not morphological surrogates for behavior (Wenzel 1992: 366).

Remane's criterion of position is the familiar one of a putative homology occupying the same physiological position or topography. For example, two bones are considered homologous because they occupy the same relative position in their respective organisms, even though those bones may vary in shape and size. The behavioral equivalent is the position of a behavior in a more general pattern of behavior. An example is a behavioral element within a bird's courtship ritual. Wenzel (1992: 366) cites the case of tail wagging motions of two Tilapia species that look different but are 
assumed homologous because they occur in the same place in courtship rituals. (The criterion of position is similar to Love's [this issue] criterion of organization.)

Remane’s criterion of special quality concerns the complexity and distinctness of a character. This criterion is motivated by the assumption that the more specialized a trait, the less likely that trait evolved more than once. Web weaving among spider species is a complex process involving qualitatively different steps. The web weaving behavior of different spider taxa are considered fairly unique and thus regarded as distinct behavioral homologies (Wenzel 1992: 366). Sometimes the criterion of special quality is satisfied by the distinctive function of a behavior. Ritualized behaviors, such as mating behaviors and territorial behaviors, are identified as homologous when they achieve the same unique function despite the use of different motor patterns to achieve that function (Wenzel 1992: 367; Lauder 1994).

Remane's criterion of continuity of intermediates depends on identifying an evolutionary series of behaviors on a continuum, from a simpler and more primitive state of behavior to a more complex and derived state. This is commonly done with ritualized forms of behavior, starting with a relatively simple form and connecting it to increasingly more complex forms (Wenzel 1992: 368). The assumption is that placing a series of behaviors on a gradual continuum serves as evidence that such behaviors are homologous.

Ethologists also have methods for testing the polarity of a homology. One is the biogenetic law, which is best thought of as a useful but fallible guide rather than as a strict law. According to the biogenetic law, the ontogeny of a homology becomes more complex as a homology evolves. Wenzel (1993) uses the biogenetic law to determine 
which instances of nest building behavior of wasps are more derived and which are more primitive. Trait polarity is also determined by the method of outgroup comparison (Ereshefsky 2001: 70-71). Suppose $\mathrm{x}$ and $\mathrm{x}^{\prime}$ are different states of a homology and they occur in species A and B. To determine which state is primitive, we look at a taxon closely related to A and B, the outgroup taxon, and see whether $\mathrm{x}$ or $\mathrm{x}^{\prime}$ occurs in that taxon. Whichever state occurs in the outgroup is the primitive state.

Stepping back, ethologists have a variety of empirical methods for testing behavioral homologies. Several conceptual points concerning these methods are worth highlighting. First, many of the criteria for identifying behavioral homology divorce behavioral homologies from their physical substrates. Ethological versions of Remane's criteria, for example, do not require that a behavioral homology correspond to a particular morphology or set of physical movements. Second, these criteria reflect the view that homology is a relation of sameness (or identity) and not qualitative similarity (Müller 2003; Ghiselin 2005). The criterion of position, for instance, does not require instances of a behavioral homology to be qualitatively similar, they just need to occupy the same position in a more general pattern of behavior. Third, the importance of such tests is not only to identify behavioral homologies and their polarities, but also to provide phylogenetic tests for adaptational hypotheses concerning behavior.

\section{Psychological Categories as Homologies}

Before discussing the lessons ethology offers for the study of psychological categories as homologies, two clarificatory remarks are in order. Often the investigation of psychological categories as homologies focuses on homologies among humans and 
existent non-humans organisms, usually primates and sometimes mammals (see Atran 2005 for a recent summary). Yet the programme of investigating psychological categories as homologies also studies homologies among Homo sapiens and extinct species in the genus Homo, as well as homologies that occur just within Homo sapiens. This paper makes no claim about the appropriate taxonomic groups for discovering psychological homologies. Whether a psychological category is a homology within Homo sapiens or a homology among humans and other species is an empirical question. The aim of this paper is to demonstrate that theoretical ideas and operational criteria from ethology can be fruitfully applied to psychological homologies wherever such homologies occur. Along similar lines, this paper assumes that whether a psychological category is a homology (versus a homoplasy) is an empirical question to be answered on a case-by-case basis. No universalist claim about all psychological categories is made here.

\section{Hierarchy}

Recall the hierarchical nature of behavioral homology outlined earlier. Many behavioral homologies do not bear a one-to-one relation to their underlying structures. Nonhomologous elements, such as non-homologous morphological structures and movements, underlie homologous behaviors. And, homologous elements, such as homologous genes and morphological structures, contribute to non-homologous behaviors.

Some psychological categories have a similar relation to their underlying structures. Gottlieb and Lickliter (2004: 312) write of "equifinality” and "multifinality” 
between developmental pathways and psychological traits. Equifinality occurs when different developmental pathways lead to the same psychological trait, and multifinality occurs when the same developmental factors lead to different psychological traits. An example of the former is non-doxastic fear (Griffiths 1997; Matthen 1998). Studies show that rhesus monkeys express fear differently depending on the social environment they are raised in (Griffiths 1997: 61). Similar results are found in humans. Americans and Japanese display negative emotions differently, and evidence indicates that such variation is due to being raised in different social environments (Griffiths 1997: 53-4, 159-160). In these cases a single homologous category varies in two ways: the developmental factors (here social inputs) that cause non-doxastic fear vary, and the phenotypic displays of nondoxastic fear vary. Because the hierarchical nature of homology encompasses both ontogenetic and phenotypic variation, a hierarchical approach to homology nicely captures the variation found among instances of a psychological category like nondoxastic fear.

The hierarchical perspective on homology also has resources for explaining why instances of non-doxastic fear are instances of a single category. Following Müller (2003) and Ghiselin (2005), homology is a relation of sameness and not qualitative similarity. That sameness is phylogenetic sameness. Variable instances of a psychological homology are instances of the same category because they share the same phylogenetic history. This phylogenetic perspective on psychological categories has implications for standard questions in the philosophy of mind. A central question is the relation between psychological states and their underlying physical states. Consider the often-cited philosophical example of being in pain. Do instances of being in pain reduce 
to a single corresponding physical-neurological state? The dominant answer in analytic philosophy is ‘no': pain, as a type of psychological state, is not reducible to one type of physical state but supervenes on multiple types of physical states (Bickle 2006). This phenomenon is often referred to as 'multiple realizability': a single psychological kind, such as pain or fear, is realized (manifested) by different physical kinds. According to most accounts of multiple realizability, a token of a psychological trait is reducible to a token physical state within an organism. However, there is no 'type:type reduction' between types of psychological states and types of physical states. For example, not all instances of non-doxastic fear correspond to one type of physiological state.

If a psychological category is a homology, then we have an account of that category’s multiple realizability. Instances of the same psychological category may vary in their physical substructures. They can also vary in their developmental causes. Nevertheless, they are instances of the same category because of their shared distinct phylogeny. Citing a category’s phylogeny explains why instances of a psychological trait belong to the same category. Furthermore, if we study the ontogeny of a psychological category we can explain why instances of the same category vary, and why they vary in a fairly stable manner. As we have seen, stable differences among instances of a homology are due to the evolution of its corresponding developmental module. Heritable differences in the genetic and non-genetic content of a developmental module are responsible for the existence of stable variation of a trait over time and at a time. Thinking of a psychological category as a homology thus brings a set of conceptual resources for understanding the multiple realizability of that category. In brief, 
phylogeny provides the identity conditions for a psychological category, and ontogeny explains stable variation among its instances.

Philosophers of mind offer various accounts of multiple realizability, and a prominent one is functionalism. According to functionalism, psychological states that mediate a similar pattern of physiological and behavioral inputs and outputs belong to the same psychological category (Bickle 2006). Functionalists are interested in the problem that such a state is solving, that is, what function a state serves. So long as instances of a psychological trait perform the same function - mediate the same patterns of behavior those instances belong to the same psychological category. However, by identifying psychological traits only by their functions, functionalists ignore the phylogeny or ontogeny of such traits. In fact, functionalists allow that instances of the same psychological category may not be historically related (for example, functionalists allow that the pain felt by humans and hypothetical extraterrestrial aliens are instances of the same psychological category). Functionalism and homology thinking offer different ways of investigating psychological categories. Functionalism focuses on the function or design problem of a psychological category; homology thinking examines the phylogeny and ontogeny of a psychological category.

In a series of writings, Griffiths (1994, 1997, 2007) and Matthen (1998, 2000, 2002, this issue) argue that homology thinking is a better way to investigate psychological categories than functionalism. Homology thinking is a general approach in biology for understanding traits. Homology thinking analyzes traits in terms of their common ancestry, whereas analogy thinking analyzes traits by studying their similarities independent of their ancestry. Consider the contrast between the homology category bird 
wing and the analogy category bird wing + insect wing. Bird wings are descended from a pair of dinosaur forelimbs and are homologous; whereas bird wings and insect wings have independent origins and are analogous. Suppose we want to understand the properties of wings. An analogy-based approach studies the general design properties of organic wings. It studies what properties are needed for flight (being aerodynamic, having a certain rigidity, and so on); and it explains the presence of those properties as a result of the function of flying. A homology-based explanation, in contrast, explains the various properties of organic wings by citing the different phylogenetic and ontogenetic factors that affect distinct lineages of wings.

In many cases, homology-based explanations are preferable to analogy-based explanations. Suppose we want to know why bird wings come in single pairs and insect wings come in double pairs. Analogy-based thinking about the general properties needed for flight will not answer that question. Instead we need to cite the distinct evolutionary histories of bird wings and insect wings. Bird wings are derived from a pair of dinosaur forelimbs. Insect wings are derived from the gills of crustaceans, which have multiple segments and were reduced to two pairs of segments in insects (Carroll 2005: 178ff). Consider another example. Suppose we want to know why penguin wings perform well as paddles. Citing the evolutionary history of penguins provides a richer explanation of the aquatic abilities of penguin wings than citing the properties needed for winged flight (Tudge 2000: 495).

The virtues of investigating a trait as a homology rather than an analogy are generic. Griffiths $(1994,1997,2007)$ and Matthen $(1998,2000)$ argue that a phylogenetic approach to psychological categories provides a better basis for explaining 
the properties of those categories than functionalism. Indeed, citing the phylogenetic history of a psychological category, and the ontogenetic factors within that phylogeny, provides a richer explanation of that category than a mere analogy-based, functional explanation. Consider the example of fear. Attending to the different developmental factors that affect instances of non-doxastic fear explains the different expressions of fear within Homo sapiens. It also explains the different expressions among primate and mammalian species. Those different developmental factors, often cultural differences, are themselves bound by distinct histories. More speculatively, if doxastic fear is a less inclusive phylogenetic unit within non-doxastic fear, then a homology-based explanation may explain the differences between those two types of fear.

Homology-based explanations of psychological traits are also more robust than adaptationist accounts offered by some sociobiologists and evolutionary psychologists (Griffiths 1997; Matthen 2002; Richardson 2007). Evolutionary psychologists focus on the selective advantage of psychological traits and analyze such traits as design problems. They use a form of reverse engineering to infer the evolutionary problems such traits were selected to solve. Despite their interest in the evolutionary basis of psychological traits, evolutionary psychologists pay little if any attention to the ontogeny or phylogeny of such traits. A homology approach to psychological categories offers a richer account of such categories because it focuses on more than just the adaptive advantage of such categories. If a psychological category is a homology, then such categories come with phylogenetic histories and ontogenetic causes. As we have seen, phylogeny causes variable instances of a psychological trait to be the same type of trait, and ontogenetic factors cause stable variation among instances of that trait. Incorporating a homology 
approach in adaptational accounts of psychological traits can only strengthen such accounts.

Stepping back, the hierarchical nature of homology emphasizes variability among instances of a homology. Consequently, a hierarchical approach to homology is wellsuited to account for the variability within a psychological category. On the one hand, studying psychological categories as homologies provides identity conditions for such categories in terms of phylogeny. On the other hand, thinking of psychological categories as homologies explains stable variation in terms of heritable ontogenetic modules. Homology thinking thus provides an explanation for the phenomenon of multiple realizability. Arguably that explanation is better than those offered by functionalist and mere adaptationist accounts.

\section{Ontogeny}

As we saw earlier, the ontogenetic turn in ethology began with Lehrman's (1953) critique of Lorenz and Tinbergen's commitment to nativism. Lehrman's work offered two lessons for ethology. First, behaviors are not simply the result of genetic inheritance but are the result of interactions between gene products (prezygotic or postzygotic) and the environment (Lehrman 1953: 26; Bateson 2003: 12). Second, early ethologists studied behavioral traits in a "purely taxonomic way" (Lehrman 1953: 35). Lehrman argued that a proper understanding of behavioral homologies also requires studying the ontogeny of behavior.

Lehrman's lessons for ethology apply to the study of psychological traits as homologies. Consider the explanations offered by evolutionary psychologists to explain 
psychological traits. Psychological traits are caused by mental modules. Such modules, according to evolutionary psychologists, contain "a priori knowledge” (Tooby and Cosmides 1992: 104) that "is specified by our genetic program" (Pinker 1997: 21). Furthermore, the psychological traits caused by mental modules are "developmentally timed to appear, disappear, or change operation to mesh with the changing demands of different age-specific tasks” (Tooby and Cosmides 1992: 81-82). The picture evolutionary psychologists give of psychological traits is reminiscent of Lorenz and Tinbergen’s view that behavioral homologies are encoded in genes and released by environmental cues (Johnston 2001; Alcock 2003; Burkhardt 2005). While ethologists have given up nativist views concerning behavior, many prominent psychologists still hold such views concerning psychology.

As mentioned in the previous subsection, evolutionary psychologists study traits as design problems. They focus on the adaptive advantage a trait does (or did) confer on an organism, and they pay little attention to the ontogeny of such traits. Similarly, functionalists in cognitive science and philosophy focus on what function a psychological state is supposed to perform, not its ontogeny. Just as studying the ontogeny of behavior improves our understanding of behavioral homologies, studying the ontogeny of psychological traits enhances our understanding of psychological categories. Consider the case of non-doxastic fear. Rhesus monkeys express fear differently depending on the social environment they are raised in. Humans also vary in how they express fear, again depending on the culture they are raised in. A proper understanding of variation among instances of non-doxastic fear cites the different developmental environments organisms are brought up in. More generally, citing ontogenetic factors is important in explaining 
variation (and similarity). Purely adaptationist and functionalist explanations miss that type of explanation.

By focusing on the ontogeny of psychological traits we can also see a problem with the nativism of evolutionary psychology. Here, again, we follow in Lehrman's footsteps. Evolutionary psychologists maintain that psychological traits are caused by genetic programmes that are triggered by independent environments. Yet at least some environmental factors that contribute to the development of psychological traits are not independent of the organisms they affect. For example, the expression of non-doxastic fear in humans varies according to the culture one is raised in. Humans construct their cultures, so some of the developmental factors that affect displays of fear in humans are human constructed. The same is true of other organisms. There are countless examples of social structures among groups of primates that affect the development of psychological or behavioral traits within primates. Such examples disconfirm the nativist view that psychological traits are the result of preset programs triggered by independent environments. Such environments are not independent of the species they affect because they are constructed by members of that species.

The poverty of genetic-nativist approach to psychology is nicely highlighted by Jablonka and Lamb’s (2005, Chapter 5) taxonomy of inheritance systems for behavior. They cite three such systems: transfer of behavior-influencing substances; imitative learning; and, non-imitative social learning. The first category contains genetic material, as well as chemicals ingested by a mother. Imitative learning includes vocal imitation by birds and other organisms. Non-imitative social learning includes birds learning how to open milk bottles and university students learning calculus. In Jablonka and Lamb’s list 
of behavioral inheritance, genetic material is just one component of one category. By attending to the ontogeny of behavioral and psychological traits we get a more robust account of behavioral inheritance. This particularly applies to humans where nonimitative learning is a pervasive factor in the development of psychological traits. As many philosophers and scientists point out, the existence of human language and our ability to store information makes human cultural evolution unique (Boyd and Silk 2000: 608-612; Sterelny 2003: 171ff). Our species’ unique cultural evolution has brought about complex social environments that significantly affect the development of our psychological traits (Sterelny 2003: 172-3; Jablonka and Lamb 2005: 213). An approach to psychological categories that sees psychological traits as preset by genes and triggered by an independent environment misses much in the ontogeny of human psychology.

Stepping back, we have seen that ethology offers two general lessons for studying psychological categories as homologies. In the previous subsection, we saw that investigating the phylogeny of psychological traits provides a better understanding of those traits than studying them from a purely adaptationist or functionalist perspective. In this subsection, we have seen that attending to the ontogeny of psychological traits provides a richer understanding of those traits than studying them from a purely adaptationist or functionalist perspective.

\section{Operational Criteria}

The third lesson from ethology concerns methods for testing psychological homologies. Sociobiologists and evolutionary psychologists posit hypotheses concerning the adaptive advantage of a psychological category. Those hypotheses are often posited and tested 
using analogy thinking. A psychological trait is treated as a design problem and two questions are the focus of inquiry: What function does the trait serve? What adaptive advantage does that function confer? Testing is often done by a hypothetical analysis of the usefulness of a trait, followed by a demonstration of its universality within humans (or some other taxon). Griffiths (1996), Matthen (2002), Richardson (2007), and others argue that such analogy-based testing only weakly discriminates among adaptational hypotheses. They suggest that the adaptive hypotheses of evolutionary psychology and sociobiology should be tested against phylogenetic evidence. Griffiths (1994) and Matthen (2002) raise a similar point about functionalist accounts of psychological traits. They suggest that functionalist accounts of psychological categories could be better tested if such hypotheses were restricted to those that posit homologies. Such hypotheses could then be subjected to an array of phylogenetic tests.

As we saw earlier, ethologists employ a wide range of phylogenetic methods for testing behavioral homologies. Those tests fall into three categories: testing whether a trait is a homology or a homoplasy; testing the polarity of a homology; and testing an adaptive hypothesis concerning a homology. If adaptational hypotheses concerning psychological traits are too easy to posit, then phylogenetic tests from ethology can be used to constrain which hypotheses to posit. In addition, phylogenetic tests can be used to more strongly confirm those adaptive hypotheses that are posited.

One concern, though, is the applicability of such phylogenetic tests to psychological traits. While Richardson suggests that adaptational hypotheses concerning psychological traits should be tested against the phylogenetic record, he is pessimistic about our access to that record (2007: 394ff). Often we lack empirical information about 
a trait's phylogeny and its past selective environment. That may be true, but anthropologists are more optimistic about obtaining historical information concerning the evolution of human psychological traits (Boyd and Silk 2000, Atran 2005). Nevertheless, it is noteworthy that ethological versions of Remane’s (1952) and Patterson’s (1982) tests for homology do not rely on historical data. Remane’s criteria of position and special quality distinguish behavioral homologies from homoplases in contemporary organisms. Patterson’s (1982) conjunction criterion can be applied using information about contemporary organisms. (Here's how it works. Suppose traits $\mathrm{x}$ and $\mathrm{x}^{\prime}$ are found in two species and are thought to be different states of the same homology. According to the conjunction test, if $\mathrm{x}$ and $\mathrm{x}^{\prime}$ occur in a single organism then that discovery disconfirms their being homologous.) Patterson's criterion of congruence can construct cladograms using data from contemporary organisms. Finally, the methods of outgroup comparison and the biogenetic law, discussed earlier, can test a trait's polarity using extant organisms.

The lesson for studying psychological categories as homologies is that regardless of our access to the historical record, an array of phylogenetic methods exists for testing adaptational hypotheses. Couple these with the various comparative methods discussed earlier and we see that ethology offers a robust store of methods for testing psychological categories as homologies. These phylogenetic tests increase the number of methods for testing adaptational and functionalist hypotheses well beyond the standard tests of design analysis and cross-cultural comparison.

Another benefit of applying ethological criteria to psychological categories is that such criteria are well suited for the variability of psychological categories. As we saw 
earlier, one reason that ethologists applied Remane's criteria to behavioral homology was their desire not to reduce behavior to morphological structures and movements. Ethologists believe that behavioral homologies are traits in themselves, and that instances of the same behavioral homology may or may not have the same type of physical substructure. The phenomenon of multiple realizability requires a similar approach to testing psychological categories, and Remane’s criteria nicely fills that need. Remane’s criteria provide methods for testing psychological homologies as psychological homologies, independent of their particular morphological and neurophysiological substructures. Though a token psychological state may correspond to token physical state, ethological criteria do not require any type:type identity between psychological states and physical states.

We have also seen that the criteria for identifying a behavioral homology do not require tokens of a behavior to be similar. Instances of the same behavior need to satisfy relational criteria such as position in a behavioral pattern, uniqueness in function, or continuity in an evolutionary sequence. These and other criteria for identifying behavioral homology provide methods for identifying variable but homologous psychological traits. Varying instances of non-doxastic fear, for example, belong to the same category if they occupy the same position in a more general pattern of behavior. And, if instances of doxastic and non-doxastic fear form an evolutionary sequence then we would have evidence that they are homologous. 


\section{Conclusion}

In summary, the lessons from ethology for studying psychological categories are many.

One set of lessons concerns identifying and testing psychological homologies.

Ethological criteria for identifying homologies provide numerous methods for testing adaptational and functionalist hypotheses concerning psychological traits. Moreover, such criteria are designed to identify the same psychological homology despite the occurrence of qualitative and substructure variation. Other lessons from ethology concern our understanding of psychological homology. Lorenz and early ethologists taught us the importance of paying attention to the phylogeny of such homologies. Lehrman and recent ethologists have taught us the importance of studying their ontogeny. Ethology contains a rich store of theoretical ideas and operational criteria that can be applied to psychological homologies. Incorporating such ideas in adaptationist and functionalist approaches to psychology would improve those approaches.

\section{Acknowledgements}

My thanks to Ingo Brigandt, David Buller, Stephen Downes, Paul Griffiths, David Hull, Alan Love, and Elliott Sober for their very helpful comments on an earlier draft of this paper. Financial support was provided by the Social Sciences and Humanities Research Council of Canada and the Calgary Institute for the Humanities. 


\section{References}

Abouheif, E.: 1999, “Establishing Homology Criteria for Regulatory Gene Networks: Prospects and Challenges” in Bock, G.R. and Cardew, G. (eds.): 1999, Homology. New York: John Wiley, pp. 207-221.

Alcock, J.: 2003, “A Textbook History of Animal Behavior”, Animal Behavior 65:3-10.

Atran, S.: 2005, “Adaptationism for Human Cognition: Strong, Spurious or Weak?”, Mind and Language 20: 39-67.

Bateson, P.: 2001, “Where Does Our Behavior Come From?”, Journal of Biosciences 26: 561-570. 2003, “The Promise of Behavioral Biology”, Animal Behavior 65: 11-17. 2004, “The origins of human differences”, Daedalus, 36-46.

Bickle, J.: 2006: “Multiple Realizability”, in Zalta, E. (ed.), The Stanford Encyclopedia of Philosophy. http://plato.stanford.edu/entries/multiple-realizability.

Bock, G.R. and Cardew, G. (eds.): 1999, Homology. New York: John Wiley.

Boyd, R. and Silk, J.: 2000, How Humans Evolved, Second Edition. New York: Norton.

Brigandt, I.: 2003, Homology in Comparative, Molecular, and Evolutionary Developmental Biology: The Radiation of a Concept, Journal of Experimental Zoology (Molecular and Developmental Evolution) 299B, 9-17.

Burian, R.: 1992, “Adaptation: Historical Perspectives”, in Keller, E. and Lloyd, E. (eds.), Keywords in Evolutionary Biology. Cambridge, MA: Harvard University Press, pp. 7-12.

Burkhardt, R.: 2005, Patterns of Behavior: Konrad Lorenz, Niko Tinbergen, and the Founding of Ethology. Chicago: University of Chicago Press. 
Carroll, S.: 2005, Endless Forms Most Beautiful: The New Science of Evo Devo. New York: Norton.

Cracraft, J.: 2005, "Phylogeny and Evo-Devo: Characters, Homology, and the Historical Analysis of the Evolution of Development”, Zoology 108: 345-356.

Ereshefsky, M.: 2001, The Poverty of the Linnaean Hierarchy: A Philosophical Study of Biological Taxonomy. Cambridge: Cambridge University Press.

Ghiselin, M.: 2005, “Homology as a Relation of Correspondence between Parts of Individuals”, Theory in Biosciences 124: 91-103.

Gottlieb, G.: 2001, “A Developmental Psychobiological Systems View: Early Formulation and Current Status”, in Oyama, S., Griffiths, P.E., Gray, R. D. (eds.), Cycles of Contingency: Developmental Systems and Evolution. Cambridge, Mass.: MIT Press, pp. 41-54.

Gottlieb, G. and Lickliter, R.: 2004, “The Various Roles of Animal Models in Understanding Human Development”, Social Development 4: 311-325.

Greene, H.: 1994, “Homology and Behavioral Repertories”, in Hall, B. (ed.), 1994, Homology: The Hierarchical Basis of Comparative Biology. San Diego: Academic Press, pp. 370-393. 1999, "Natural History and Behavioral Homology”, in Bock, G.R. and Cardew, G. (eds.), Homology. New York: John Wiley, pp. 173-182.

Griffiths, P. E.: 1994, “Cladistic Classification and Functional Explanation”, Philosophy of Science 61:206-227. 1996, “The Historical Turn in the Study of Adaptation”, British Journal for the Philosophy of Science 47: 511-532. 
1997, What Emotions Really Are: The Problem of Psychological Categories.

Chicago: University of Chicago Press.

2007, “Evo-Devo Meets the Mind: Towards a Developmental Evolutionary

Psychology”, in R. Sansom and R. Brandon (eds.), Integrating Evolution and

Development: From Theory to Practice. Cambridge, Mass.: MIT Press.

Hall, B. K.: 2003, “Descent with Modification: The Unity Underlying Homology and Homoplasy as Seen Through an Analysis of Development and Evolution”, Biological Review 78:409-433.

(ed.), 1994, Homology: The Hierarchical Basis of Comparative Biology. San Diego: Academic Press.

Jablonka E., and Lamb, M.: 2005, Evolution in Four Dimensions: Genetic, Epigenetic, Behavioral, and Symbolic Variation in the History of Life. Cambridge, MA: MIT Press.

Johnston, T.: 2001, “Toward a Systems View of Development: An Appraisal of Lehrman’s Critique of Lorenz”, in Oyama, S., Griffiths, P.E., Gray, R. D. (eds.), Cycles of Contingency: Developmental Systems and Evolution. Cambridge, Mass.: MIT Press, pp. 15-24

Lauder, G.: 1994, “Homology, Form, and Function”, in B. Hall (ed.), Homology: The Hierarchical Basis of Comparative Biology. San Diego: Academic Press, pp. 151196.

Lehrman, D.: 1953, “A Critique of Konrad Lorenz’s Theory of Instinctive Behavior”, Quarterly Review of Biology 28:337-363. Reprinted in Oyama, S., Griffiths, P.E., 
Gray, R. D. (eds.), Cycles of Contingency: Developmental Systems and Evolution. Cambridge, Mass.: MIT Press, pp. 25-40.

Matthen, M.: 1998, "Biological Universals and the Nature of Fear”, Journal of Philosophy XVC (3):105-132. 2000, “What is a Hand? What is a Mind?”, Revue Internationale de Philosophie 214:653-672. 2002, "Human Rationality and the Unique Origin Constraint”, in Ariew, A., Cummins, R., and Perlman, M. (eds.), Functions: New Essays in the Philosophy of Psychology and Biology. New York: Oxford University Press, pp. 341-372. Müller, G.: 2003, “Homology: The Evolution of Morphological Organization”, in Müller, G. and Newman, S. (eds.), Origination of Organismal Form: Beyond the Gene in Developmental and Evolutionary Biology. Cambridge, MA: MIT Press, pp. 52-69 Patterson, C.: 1982, “Morphological Characters and Homology”, in Joysey, K. and A. Friday (eds.), Problems of Phylogenetic Reconstruction. London: Academic Press, pp. 21-74.

Pinker, S.: 1997, How the Mind Works. New York: Norton. Raff, R.: 1996, The Shape of Nature: Genes, Development, and the Evolution of Animal Form. Chicago: Chicago University Press.

Remane, A.: 1952, Die Grundlagen des natürlichen Systems, der vergleichenden Anatomie und der Phylogenetik. Leipzig: Geest und Portig. Richards, R.: 1987, Darwin and the Emergence of Evolutionary Theories of Mind and Behavior. Chicago: Chicago University Press. 
Richardson, R.: 2007, “The Adaptive Programme of Evolutionary Psychology”, in Thagard, P. (ed.), The Philosophy of Psychology and Cognitive Science. Amsterdam: Elsevier, pp. 369-428.

Sterelny, K.: 2003, Thought in a Hostile World: The Evolution of Human Cognition. Oxford: Blackwell.

Sterelny, K. and Griffiths P.: 1999, Sex and Death: An Introduction to Philosophy of Biology. Chicago: Chicago University Press.

Striedter, G. and Northcutt, G.: 1999, "Biological Hierarchies and the Concept of Homology”, Brain, Behavior and Evolution 38: 177-189.

Tooby, J. and Cosmides, L.: 1992, “The Psychological Foundation of Culture”, in Barkow, J., Cosmides, L., and Tooby, J. (eds.) The Adapted Mind: Evolutionary Psychology and the Generation of Culture. New York: Oxford University Press, pp. 19-136.

Tudge, C.: 2000, The Variety of Life. Oxford: Oxford University Press.

Wagner, G.: 1996, "Homologues, Natural Kinds and the Evolution of Modularity”, American Zoologist 36: 36-43. 1999, “A Research Programme for Testing the Biological Homology Concept”, in Bock, G.R. and Cardew, G. (eds.): 1999, Homology. New York: John Wiley, pp. 125-134. 2007, “The Developmental Genetics of Homology”, Nature Reviews Genetics 8: 473-479. (ed.), 2001, The Character Concept in Evolutionary Biology. London: Academic Press. 
Wenzel, J.: 1992, “Behavioral Homology and Phylogeny”, Annual Review of Ecology and Systematics 23: 361-381. 1993. “Application of the Biogenetic Law to Behavioral Ontogeny: A Test Using Nest Architecture in Paper Wasps”, Journal of Evolutionary Biology 6: 229-247. 